\title{
Les images des ennemis dans la perception des conquérants de l'Europe (1805-1812)
}

The Image of Enemies in the Perception of the Conquerors of Europe (1805-1812)

\section{Maya Goubina}

\section{(2) OpenEdition \\ 1 Journals}

Édition électronique

URL : https://journals.openedition.org/ahrf/12599

DOI : 10.4000/ahrf.12599

ISSN : 1952-403X

\section{Éditeur :}

Armand Colin, Société des études robespierristes

\section{Édition imprimée}

Date de publication : 1 septembre 2012

Pagination : 81-93

ISBN : 978-2-200-92761-5

ISSN : 0003-4436

\section{Référence électronique}

Maya Goubina, «Les images des ennemis dans la perception des conquérants de l'Europe

(1805-1812) », Annales historiques de la Révolution française [En ligne], 369 । juillet-septembre 2012, mis en ligne le 01 septembre 2015, consulté le 24 avril 2022. URL : http://journals.openedition.org/ahrf/ 12599 ; DOI : https://doi.org/10.4000/ahrf.12599 


\title{
LES IMAGES DES ENNEMIS DANS LA PERCEPTION DES CONQUÉRANTS DE L'EUROPE (1805-1812)
}

\author{
Maya GOUBINA
}

\begin{abstract}
Les guerres napoléoniennes ont amené des centaines de milliers de combattants français dans les différents « ailleurs » vers la rencontre avec les Autres. Dans cet article, nous avons entrepris l'analyse comparative des écrits (la correspondance, les carnets et les journaux) des militaires français qui ont participé aux différentes campagnes impériales en 1805-1812 afin de reconstituer les éléments de la perception qu'ils eurent des Prussiens et des Russes, des Espagnols, des Portugais et des Autrichiens.
\end{abstract}

Mots-clés : voyage, pittoresque, ego-documents, perceptions, géographie, caractères nationaux, culture matérielle.

« Le temps était clair et doux,

on entendait chanter les rossignols

et le paysage invitait plutôt à une fête champêtre

qu'à des combats meurtriers $»^{1}$.

« Il y a neuf mois, je me baignais dans les eaux du Memel ;

aujourd'hui j'ai affronté les flots du Tage $»^{2}$.

L'impact de l'époque napoléonienne dans l'évolution des perceptions mutuelles des nations européennes et, notamment, dans la formation des représentations françaises de l'Autre attire de plus en plus l'attention

(1) François PILS, Journal de marche du grenadier Pils (1804-1814), Paris, P. Ollendorff, 1895 , p. 42.

(2) Maurice de TASCHER, Notes de campagnes (1806-1813), Châteauroux, Société d'Imprimerie, d'Édition et des Journaux du Berry, 1932, p. 88. 
des spécialistes. Les guerres napoléoniennes amenaient des centaines de milliers de Français dans les différents « ailleurs » à la rencontre des armées adverses, mais aussi avec la population civile.

« Arraché par la conscription à l'horizon étroit de son village natal, le fils de paysan voyait défiler devant lui les plaines monotones de l'Allemagne, les montagnes arides d'Espagne, les steppes glacées de Russie ; il découvrait Vienne, Berlin et Moscou, Venise et Madrid. Nul voyageur du $\mathrm{XVIII}^{\mathrm{e}}$ siècle n'a parcouru un itinéraire européen comparable à celui du capitaine Coignet $»^{3}$.

Nous nous proposons d'analyser l'image de l'ennemi ou, plutôt, les images des ennemis, qui se sont formées chez les soldats et officiers napoléoniens pendant la période choisie. Cette dernière englobant plusieurs campagnes, nous effectuerons une analyse comparative des perceptions françaises des différents peuples ${ }^{4}$. La sensibilité (parfois véritablement romantique) des « voyageurs malgré eux »-les participants des campagnes impériales - au pittoresque des terres inconnues est un thème déjà éprouvé ${ }^{5}$.

Les limites chronologiques correspondent à la période où la présence massive des Français était, d'une part, indissociablement liée avec le nom d'un seul personnage historique - l'empereur Napoléon. D'autre part, pendant cette période cette présence française dans les pays européens était le fruit de la domination politique et militaire impériale, même si la guerre en Espagne en représentait une faille importante. Ce n'est, en effet, que la campagne de Russie de 1812 qui constituera le réel tournant de la fortune militaire napoléonienne sur terre et qui entraînera la défaite du régime.

Les objectifs de ce travail imposent certains critères de choix de documents. En premier lieu, nous utiliserons uniquement les textes qui sont contemporains des événements : la correspondance, les journaux intimes et les carnets de route ${ }^{6}$. Nous avons exclu de notre base de travail tous les écrits

(3) Jean TUlARD, «Préface» dans Jean TUlARD, Nouvelle bibliographie critique des mémoires sur l'époque napoléonienne écrits ou traduits en français, Genève, Droz, 1991, p. 7.

(4) La guerre de la $3^{\mathrm{e}}$ coalition (1805), la campagne de Prusse (1806), la première guerre de Pologne (1806-1807), la guerre d'Espagne (1807-1814), la campagne d'Autriche (1809), la campagne de Portugal (1807-1808) et la campagne de Russie (1812).

(5) Voir les travaux de nos collègues russes (Vladimir. N. Zemcov, Nicolaï V. Promyslov) et français (Marie-Pierre Rey, Natalie Petiteau, etc.) ainsi que nos articles et notre thèse de doctorat disponible en ligne sur le site de l'Université Paris IV (http://www.theses.paris4.sorbonne.fr/these goubina/paris4/2007/these_goubina/html/index-frames.html).

(6) Le travaii présent se dîrférencie en ceia de îarticie de îatailie Petiteau et nous verrons comment la limitation du corpus des sources aux textes strictement contemporains permet de nuancer le tableau brossé par l'historienne. En outre, nous utiliserons plus largement les égo-documents relatant la campagne de Russie. Voir Natalie PETITEAU, « Les voyages des hommes de la Grande Armée : de la 
rédigés a posteriori même si les notes prises au jour le jour leur avaient servi de base. Afin d'essayer de capter des traces de formation des perceptions, il est indispensable d'éliminer les mémoires bien postérieurs aussi bien que les souvenirs rédigés seulement un ou deux ans après les événements décrits. En effet, les changements politiques ayant été rapides et importants, il est fort probable que les Français pouvaient décrire leurs propres impressions différemment avant et après la chute du régime napoléonien. En deuxième lieu, nous avons naturellement limité notre corpus aux écrits des Français en mettant de côté ceux de leurs alliés, y compris francophones, plus ou moins forcés de servir dans les troupes napoléoniennes. En troisième lieu, nous avons exclu les notes des prisonniers : leur statut et la durée de leur présence sur le sol étranger réduisent les possibilités de saisir les impressions et perceptions instantanées.

Il est, enfin, important de rappeler que plusieurs circonstances influençaient l'attitude des participants des guerres napoléoniennes. En premier lieu, il s'agit de la position des auteurs dans la hiérarchie militaire. En deuxième lieu, il faut tenir compte de la nature de leurs responsabilités et de leurs fonctions dans les armées. Il est évident que le regard d'un soldat de ligne ne sera pas le même que celui d'un chirurgien ou d'un pharmacien militaire, alors que la perception des événements et des réalités sera encore différente chez un commissaire de guerre ou chez un aide-de-camp, etc. En troisième lieu, les circonstances d'ordre purement militaire (périodes de siège ou offensives) et la position des régiments des auteurs (participation dans telle ou telle bataille ou attente dans la réserve, etc.) jouent un rôle important dans la formation de l'état d'esprit des auteurs des textes étudiés. Enfin, les circonstances d'ordre personnel (avant tout l'état de santé, mais aussi la situation familiale, etc.) ont leur impact sur la perception par les militaires des événements et des réalités.

\section{Capacités et disponibilités pour la découverte de l'Autre}

La découverte de l'Autre, surtout dans les conditions spécifiques des opérations militaires, exige non seulement une disponibilité d'esprit et une curiosité certaines, mais aussi des capacités bien précises. Notons que le gouvernement impérial, dans son souci d'augmenter les chances de conquête, favorisait l'aspect «découverte» de ses campagnes : la 
publication des dictionnaires de langues en témoigne. La consultation des catalogues de la Bibliothèque Nationale de France permet de voir les résultats de cette production, alors que les textes des égo-documents en illustrent l'usage. Pereuse note : « $\mathrm{j}$ 'avais toujours dans ma poche un vocabulaire espagnol qui me permettait de me faire comprendre $»^{7}$.

Plusieurs militaires signalent plus ou moins explicitement l'importance de la maîtrise de la langue des contrées « visitées ». Et si H.-J.-V. de Saint-Simon souligne cet aspect plutôt du point de vue des circonstances sa maîtrise de l'espagnol lui a plusieurs fois permis d'éviter le pire ${ }^{8}-$ ses compagnons d'armes affichent une attitude qui les rapproche des voyageurs curieux. Chaque entrée dans un nouveau pays est signalée dans le journal de Choderlos de Laclos par le vocabulaire des mots les plus usités avec leur traduction en idiome local. Le vocabulaire polonais contient même des expressions et de courtes phrases ${ }^{9}$. Lors de la campagne de Prusse en 1806, M. de Tascher profite, quant à lui, des moments de calme... pour apprendre la langue du pays. « Je coule ici des jours bien tranquilles [...] la pêche et l'étude de l'allemand partagent mon temps qui s'écoule sans que je m'en aperçoive $»^{10}$. Deux ans plus tard - en Espagne - il regrettera de ne pas pouvoir poser des questions en visitant la ville de Burgos faute de savoir l'espagnol ${ }^{11}$. Alors qu'en $1812 \mathrm{~J}$. Bréaut des Marlots essaie de profiter de sa double chance de tomber dans une maison non désertée et habitée par des francophones afin de pallier son ignorance du russe. «J'étais tombé dans une maison française d'origine [...] Je priai ces dames [...] de me mettre un peu au courant des usages russes $\gg^{12}$. En outre, nombreux sont ceux qui expliquent dans leurs notes la signification des noms des lieux géographiques traversés.

Le complexe de supériorité est, bien entendu présent dans les écrits des Français, parfois sous une forme très explicite : le luxe et l'élégance

(7) PEREuSE, « Campagne de Portugal (1810-1811)», Revue rétrospective, 1889, t. X, p. 2.

(8) Henri Jean Victor de SAINT-SIMON, « Carnet de campagne du duc de Saint-Simon », Carnet Historique et Littéraire, 1899, t IV, p 244- 246 ; « Campagne d'Espagne de 1808-1809 »Ibid, 1899, t IV, p. 439-441.

(9) Voir Étienne Fargeau CHODERLOS DE LAClos, Le fils de Laclos. Carnets de marche du commandant Choderlos de Laclos, an XIV-1814, Lausanne, Payot et $\mathrm{C}^{\mathrm{ie}}$; Paris, Fontemoing et $\mathrm{C}^{\mathrm{ie}}$, 1912, p. 71-73. L'auteur de ces carnets est le fils de l'auteur des célèbres Liaisons dangereuses.

(10) Maurice de TASCHER, op. cit., p. 23-24. Voir aussi à propos de ses efforts dans l'étude de l'allemand ; Ibid, p. 38.

(11) Ibid, p. 75.

(12) Jean BRÉAUT DES MARLOTS, « Lettre d'un capitaine de cuirassiers sur la campagne de Russie » dans Jean BRÉAUT, Lettre d'un capitaine de cuirassiers sur la campagne de Russie; Pierre Paul DENNIÉE, Itinéraire de l'empereur Napoléon pendant la campagne de 1812, Paris, La Vouivre, 1997 , p. 8. 
des cafés viennois seraient « au moins à un siècle en arrière de Paris $»^{13}$. Cela n'empêche cependant pas les conquérants de faire preuve de curiosité et d'une bonne capacité d'observation, voire d'objectivité. Ainsi, le même Choderlos de Laclos atteste que la ménagerie offre de meilleures conditions de vie aux animaux à Schönbrunn qu'à Paris et, plus loin, il confirme qu'à Tilsit les villages des civils sont également dévastés aux alentours du camp russe et de celui français ${ }^{14}$.

Soulignons, enfin, l'envie de découvrir et de décrire manifestée par les conquérants napoléoniens. La richesse de leurs textes en est la première preuve. En outre, certains militaires expriment leur déception quand les circonstances ne leur permettent pas de consacrer autant de temps qu'ils voudraient à la « visite » des lieux ; d'autres le précisent pour expliquer la brièveté de leurs notes. «Laisser la jolie petite ville d'Ips située au bord du fleuve sans la visiter fut un peu cruel pour moi, mais il fallut se conformer aux circonstances $»^{15}$. Plusieurs sont, donc, conscients du rôle d'intermédiaires culturels qu'ils peuvent quasiment jouer auprès de leurs contemporains qui liront leurs lettres ou leurs carnets de routes. « Il est inutile que j'entreprenne de faire la description de cette capitale [Vienne], attendu qu'elle est déjà assez connue ${ }^{16}$.

\section{Contrées « visitées »}

\section{Géographie : temps, climat, saisons, paysages et routes}

Les remarques sur le climat, le temps et les routes sont parmi les plus fréquentes dans les textes des soldats et officiers napoléoniens, qu'il s'agisse de l'effervescence des offensives ou des marches intermédiaires, voire des périodes de repos dans les bivouacs et dans les villes. Même si les indications sur ces sujets étaient, à l'origine, destinées à illustrer les difficultés de la vie d'un soldat, leur régularité et leur nombre dépassent le cadre d'une simple chronique quotidienne de la vie des militaires ${ }^{17}$. Les

(13) Étienne Fargeau CHODERLOS DE LACLOS, op. cit., p. 38.

(14) Ibid., p. 43-44, 121.

(15) François DURIAU, «Carnet de route», Mémoires de la Société dunkerquoise pour l'encouragement des sciences, des lettres et des arts, 1907, t. 46, p. 42.

(16) Étienne Fargeau CHODERLOS DE LACLOS, op. cit., p. 37.

(17) Nous ne sommes donc pas d'accord avec l'opinion de Marie-Cécile Thoral qui qualifie ce sujet simplement de «chronique de la vie dans les bivouacs » tout en l'opposant aux écrits des voyageurs traditionnels qui auraient fourni des informations plus complètes (quel temps fait-il en général dans la contrée traversée dans une saison donnée, quel type de climat y règne ?) En effet, combien de vrais voyageurs de la même époque mentionnaient non seulement le temps qu'ils avaient eu la chance ou la malchance de subir, mais réussissaient aussi à fournir des renseignements plus larges 
participants de la campagne de Russie signalent les spécificités du climat nordique comme, par exemple, les nuits blanches inconnues en France : " nous n'avons presque pas de nuit c'est un crépuscule d'environ deux heures $\gg^{18}$. Les égo-documents permettent également de confirmer que les grands froids du mois de novembre n'ont été que l'apogée et non pas le seul exemple du fort impact que les conditions climatiques ont eu sur le déroulement des opérations militaires et, surtout, sur l'état d'esprit des Français en $1812^{19}$.

Les descriptions des paysages espagnols, portugais, mais aussi de ceux de l'Europe centrale attirent l'attention du lecteur par leur style romantique : «les gorges des roches », «des vastes plaines» et les positions « riantes» des villages remplissent les notes des militaires. Certains d'entre eux vont faire des promenades en dehors des bivouacs pour découvrir d'autres paysages moins connus. Ainsi, Choderlos et Duriau vont se rendre sur les bords de la Baltique ${ }^{20}$. Si les paysages russes inspirent moins les participants de la guerre de 1812, ces derniers sont, en revanche, impressionnés par la beauté inhabituelle des routes en Russie. Le témoignage de J. Bréaut est l'un des plus impressionnants. L'image des horreurs vues sur le champ de Borodino laisse la place dans sa lettre à l'image étrangement paisible et précise de la route menant vers l'ancienne capitale russe.

« Revenons sur la route de Moscou qui est un chef-d'œuvre de l'art. On peut marcher à dix voitures de front. De chaque côté il y a deux rangées d'arbres très hauts entre lesquels il y a un chemin pour les personnes à pied. Ces arbres ressemblent beaucoup aux saules pleureurs ; ils préservent en été des fortes chaleurs par leur ombrage hospitalier, et en hiver ils servent de guides quand la neige remplit les précipices qui sont fréquents, en confondant le ciel avec la surface de la terre $»^{21}$.

Notons, en outre, qu'en 1812 les militaires français sont amenés à éprouver par leur propre expérience la véracité de l'un des stéréotypes

sur les pays traversés ? Voir Marie-Cécile THORAL, «Sur les routes avec les hommes de Wellington : le " voyage " des militaires anglais en France en 1813-1814 », dans Nicolas BOURGUINAT, Sylvain VENAYRE (dir.), op. cit., p. 289.

(18) François Roch LEDRU DES ESSARTS, Un grand patriote sarthois méconnu : la vie de ce soldat courageux qui n'aimait que la paix, Le Mans, J.-L. Bonnéry, 1988, p. 82.

(19) Pour plus de détails à ce propos voir notre thèse de doctorat.

(20) Étienne Fargeau ChOderlos DE LaClos, op. cit., p 137 ; François DuRIAU, Mémoires, op. cit., t. 46, p. 49.

(21) Jean BRÉAUT DES MARLOTS, op. cit., p. 6. 
classiques sur la Russie : celui des espaces russes à perte de vue. Enfin, les remarques concernant la faune, la flore et l'agriculture (la nature des terres et les cultures) des territoires traversés complètent les descriptions des paysages. « Les environs de la ville sont charmants, ils sont remplis de vignobles et de petits jardins $»^{22}$.

\section{Villes}

Le succès ou la difficulté du déroulement d'une campagne militaire sont reflétés dans les égo-documents de différentes façons plus ou moins explicites. Les remarques sur la présence ou l'absence des autochtones dans les villages et les bourgs traversés est une preuve implicite de la difficulté de la campagne. En effet, ce sont les participants de la campagne de Portugal, de celle d'Espagne et de celle de Russie qui prennent rapidement l'habitude de retenir ces informations, alors que leurs compagnons d'armes qui se trouvaient en Prusse ou en Autriche ne couraient pas le risque de trouver les habitations vides. Tous les militaires sont, en revanche, unanimes dans l'application avec laquelle ils signalent toutes les étapes de leurs marches et ne manquent presque jamais d'en évoquer quelques traits caractéristiques (« jolie petite ville », « mauvais village »), sans parler des descriptions bien détaillées et développées des grandes villes connues.

L'aspect « découverte » des campagnes napoléoniennes est, en effet, surtout explicite dans les notes consacrées aux séjours dans les villes des contrées conquises. Les militaires des armées napoléoniennes décrivent l'infrastructure et l'architecture des villes, les styles de construction qui y sont adoptés. Ainsi, la ville de Dantzig est « bien bâtie d'après la méthode allemande $»^{23}$. Notons que même les notes sur le séjour dans Moscou, dont la majeure partie a disparu dans les flammes dès sa reddition, témoignent de l'effort des militaires français. Le majestueux tableau de l'embrasement de l'ancienne capitale russe leur sert surtout de preuve indiscutable de caractère viscéralement autre de la mentalité russe. «C'est la plus belle horreur que j'ai vue et verrai de ma vie [...] Les Russes sont de grands barbares $»^{24}$. En revanche, ils tâchent de décrire Moscou où plutôt de reconstruire son image d'après ce qui en restait après l'incendie. " La ville de Moscou était grande et belle, pas aussi uniformément bâtie que Paris,

(22) François Duriau, Mémoires, op. cit., t. 46. p. 59.

(23) Étienne Fargeau CHODERLOS DE LACLOS, op. cit., p 135-136.

(24) « [Lettre de] Baurvott [...] à sa femme, à Rostock, Moscou, le 29 septembre 1812 »dans Sergěj Michajlovič GORIAÏNOW [et al.], Lettres interceptées par les Russes durant la campagne de 1812, Paris, Carnet de la Sabretache, 1913, p. 58. 
mais beaucoup plus grande et ayant au moins cinq cents palais plus que Paris, des magasins immenses $»^{25}$. Si les occupants de Moscou brûlée et des villes d'Espagne gangrenées par les horreurs commises par et contre la guérilla arrivent à en rédiger des descriptions pittoresques, c'est d'autant plus le cas de ceux qui « visitent » Vienne, Berlin, etc. et qui en offrent des récits de plusieurs pages. Les théâtres et les musées, les promenades et les fortifications sont plus ou moins longuement décrits, mais aussi analysés de plusieurs points de vue : leur modernité et leur confort, leur beauté et leur emplacement, leur richesse et leur histoire. Ainsi, en notant ses observations concernant les fortifications de la ville de Wurzbourg, F. Duriau fait un aveu très intéressant qui témoigne de son objectivité : « si j'osais je dirais qu'elle est imprenable (quoique ce mot soit exclu du dictionnaire français) $»^{26}$.

Enfin, tout en rendant hommage aux lieux mémorables de la gloire des armes françaises dans les campagnes passées, les militaires des armées napoléoniennes évoquent également les lieux de gloire de leurs adversaires. «On y remarque une pyramide qui a été érigée en mémoire d'un colonel de hussards autrichiens tué dans le même endroit lors de la guerre des années VIII et IX $»^{27}$. En outre, les militaires français visitent les lieux de mémoire et d'histoire de leurs ennemis, comme, par exemple, le tombeau de Marie-Thérèse à Vienne ${ }^{28}$ ou « Frédéric-Le-Grand sur son cheval de bronze » à Berlin ${ }^{29}$.

\section{Mours, coutumes et caractère national}

Il est évident que ce sont les conditions d'une « guerre civilisée »stationnement dans les villes et les villages chez l'habitant - qui permettaient aux militaires français de mieux « découvrir» les mœurs et les coutumes locales. Les conquérants décrivent les dîners et les soirées (jeux et danses) organisés par leurs hôtes. L'extrait du carnet de J. Dumas consacré à son séjour à Vienne en hiver 1805-1806 après la bataille d'Austerlitz est particulièrement riche en témoignages de ce genre. «Vienne, le 6 [janvier]

(25) Jean BRÉAUT DES MARLOTS, op. cit., p. 8.

(26) François DURIAU, Mémoires, op. cit., t. 46, p. 45.

(27) Jacques-Louis-Laurent-Augustin VIAL, « Journal d'un mois de campagne à la Grande Armée », Carnet de la Sabretache, 1901, p. 452.

(28) Étienne Fargeau CHODERLOS DE LACLOS, op. cit., p. 46-47. Notons que Choderlos de Laclos le compare au mausolée du maréchal de Saxe qu'il a vu à Strasbourg.

(29) C.-H. LEJEUNE, « Route faite par moi, Lejeune, à compter du 15 novembre 1805 / Souvenirs du Cavalier C.-H. Lejeune du II ${ }^{\mathrm{e}}$ régiment de chasseurs à cheval (1805-1810) », Carnet de la Sabretache, 1910, p. 518. 
(jour des rois) - Grand dîner chez notre hôte ; nous y avons tiré le gâteau des rois. Les six demoiselles de Funtkirchen et autres dames y étaient invitées ; la soirée était charmante $»^{30}$. Les histoires galantes sont une autre illustration d'une sorte d' « intégration » dont bénéficiaient les conquérants. Ces derniers font également des sorties aux théâtres, dans les promenades publiques et au musée. Par conséquent, les écrits des Français contiennent de nombreuses remarques, descriptions, voire réflexions sur les mœurs et coutumes des habitants des terres allemandes et autrichiennes. D'après les uns les Allemands [sic] auraient un «caractère froid, maussade même $»^{31}$, les autres tout en leur accordant quelques « apparences » positives ${ }^{32}$ mettent en garde contre leur manque de franchise. Les autres encore font des comparaisons tout en évoquant la couleur locale : «Vienne est certainement préférable à Berlin. Dans la capitale de l'Allemagne du nord, on trouve trop de pipes et on boit trop de bière $»^{33}$. Duriau décrit les passe-temps habituels des habitants de Berlin et de ses alentours : "pendant que les femmes boivent du thé et tricotent, les hommes fument la pipe, boivent [du] café ou [de la] bière, politiquent à toute outrance $»^{34}$. Enfin, M. de Tascher est particulièrement sensible à l'esthétique vestimentaire. Son journal contient un grand nombre d'observations concernant les costumes des femmes et des hommes des contrées traversées avec, éventuellement, des remarques sur le physique des habitants. Ses notes sur la Prusse contiennent, par exemple, ses observations sur les différences entre les vêtements des femmes catholiques et ceux des femmes protestantes ${ }^{35}$.

Les contacts avec la population espagnole sont extrêmement complexes et, pourtant, les écrits des Français contiennent quelques informations sur cet ennemi. "Les Castillans sont superbes d'allure ${ }^{36}$ Saint-Simon profite, en outre, d'un moment de calme dans Madrid pour mener le train de vie « ordinaire » et va au théâtre italien à quatre reprises ${ }^{37}$.

En 1812 l'armée russe se replie sans cesse tout en appliquant la politique de la «terre brûlée », la population civile quitte, elle aussi, les villes et les villages. Cette démarche « isolationniste » réduit les possibilités

(30) Jérôme DuMAS, « Journal historique de la division des grenadiers d'Oudinot », Carnet de la Sabretache, 1910, p. 557.

(31) Jacques-Louis-Laurent-Augustin VIAL, op. cit., p. 454.

(32) Jean-Baptiste GIRAUD, Le carnet de campagne du commandant Giraud, Paris, Téqui, 1898 , p. 160-161.

(33) [Lettre du capitaine Marion, beau-frère de J.-B. Giraud, Berlin, le 25 octobre 1806] Jean-Baptiste GIRAUD, op. cit., p. 125-127.

(34) François Duriau, Mémoires, op. cit., t. 46, p. 52.

(35) Maurice DE TASCHER, op. cit., p. 14-15.

(36) Jean-Baptiste GIRAUD, op. cit., p. 256.

(37) Henri Jean Victor de SAINT-SimON, op. cit., t. IV, p. 249-250. 
de contact encore plus qu'en Espagne. Les militaires de la Grande Armée ne manquent pas de signaler l'absence de l'aspect « sociable » de la campagne de Russie. « Nous n'avons pas vu de femmes depuis les maîtresses de poste de la Pologne, mais en revanche nous sommes grand[s] connaisseurs en incendies $»^{38}$. Ainsi, en 1812 les Français sont forcés de découvrir les traits du caractère - l'opiniâtreté et la capacité de sacrifice - du peuple russe ainsi que sa mentalité autre. Les Français sont obligés de reconnaître que les Russes ont réussi à transformer cette guerre en un affrontement non pas deux empires mais deux systèmes de valeurs ${ }^{39}$. Notons, en outre, que la résistance russe et le déroulement absolument imprévu de la campagne mettent à l'épreuve le complexe de supériorité affiché par les militaires de la Grande Armée au début de l'offensive.

\section{Culture matérielle}

Pendant les campagnes dans les contrées où les habitants s'en fuyaient à l'approche de l'ennemi (la campagne de Russie en 1812, mais aussi celles d'Espagne et de Portugal ainsi que la première guerre de Pologne en 1807), les observations ethnographiques des Français concernaient plutôt la culture matérielle de l' « ailleurs » visité. Il est frappant que Giraud utilise l'image de cet « ailleurs » récemment découvert afin de mieux décrire ce qu'il vit chez lui. C'est en racontant son séjour à Paris qu'il signale avoir aussi bien dormi «que dans les lits des grands seigneurs polonais $»^{40}$ Les participants de la campagne de Russie décrivent également beaucoup les palais de la noblesse russe qui les avaient impressionnés par leurs richesses. L'ameublement, les œuvres d'art, les bibliothèques et les caves des seigneurs moscovites sont régulièrement évoqués dans les écrits des militaires de la Grande Armée. Le détail des descriptions des châteaux et palais de la noblesse russe et polonaise dépasse de loin l'intérêt premier des militaires en campagne soucieux de la question du logement. C'est quand il s'agit des habitations du simple peuple que le côté pratique et l'utilité attirent davantage l'attention des militaires français et sa remarque n'est pas du tout ironique. Cependant, ces descriptions-là témoignent, elles aussi, de l'intérêt que les militaires français portent à la découverte. Ainsi certains

(38) « [Lettre de] H. Beyle à la comtesse Daru, Moscou, le 16 octobre [1812] » Lettres interceptées [...], op. cit., p. 160.

(39) Vladimir. N. ZEMCOV, « Napoleon v Moskve [Napoléon à Mocou] », dans Francuzskij ežegodnik [Annuaire français] 2006, Moskva, 2006, p. 199-218 et en accès libre : http://annuairefr.narod.ru/statji/Zemtsov-2006.html.

(40) Jean-Baptiste GIRAUD, op. cit., p. 167. 
sont capables de percevoir l'esthétique inconnue des isbas russes. «Beauté des villages, bien alignés, menuiserie en dentelle ${ }^{41}$. D'autres observent les fonctionnalités des chaumières des montagnards portugais. « Nous y trouvâmes encore, sur des claies posées sur les poutres qui supportaient la toiture, une grande quantité de châtaignes desséchées [...] Les cheminées, dans ce pays, sont tout à fait inconnues. On a fait du feu au milieu de la pièce et la fumée se dégage à travers les tuiles creuses des toits : elle contribue ainsi à dessécher les châtaignes $»^{42}$.

Les notes des participants de la campagne de 1812 non seulement s'inscrivent parfaitement dans la tradition de la perception française de l'architecture religieuse russe, mais en constituent le chaînon manquant ${ }^{43}$. En effet, les voyageurs occidentaux des $\mathrm{XVI}^{\mathrm{e}}$ et XVII ${ }^{\mathrm{e}}$ siècles « à de rares exceptions près, n'ont pas su voir la beauté de cet art [religieux orthodoxe]. Affaire de sensibilité sans doute ; il faudra attendre le XIX ${ }^{\mathrm{e}}$ siècle pour le découvrir $»^{44}$. Ce sont précisément les écrits des militaires de la Grande Armée qui ouvrent l'époque du véritable intérêt français envers les églises orthodoxes en en offrant de nombreuses descriptions du décor extérieur et intérieur, des clochers et des croix, des icônes et des crucifix. En analysant les textes sur la Russie et en constatant le nombre important de remarques sur la présence des églises, nous nous sommes demandés si cette attitude des militaires de la Grande Armée ne s'expliquait pas par les circonstances particulièrement pénibles de la campagne. Les moyens et les petits villages russes ne possédaient pas leur église. Les clochers à l'horizon, annonçant donc la présence d'un bourg ou d'une ville, ne ranimaient-ils chez les combattants un nouvel espoir de quartiers éventuels ? La présence ou l'absence des clochers à l'horizon avait dans les conditions sévères d'une campagne difficile, une importance vitale pour les militaires de la Grande Armée. La confirmation de notre hypothèse se trouve dans les textes des participants de la campagne d'Espagne. "On voit parfaitement d'ici Saragosse et, par le nombre de clochers et de monuments élevés, on juge que c'est une grande et belle ville ${ }^{45}$

(41) Maurice de TASCHER, op. cit., p. 311.

(42) PEREUSE, art. cit., p. 19.

(43) Voir leur analyse approfondie dans notre thèse de doctorat.

(44) Michel Mervaud, «La Russie vue par les voyageurs français du XVI ${ }^{\mathrm{e}}$ et du XVII siècles » dans Michel MERVAUD, Une infinie brutalité : l'image de la Russie dans la France des XVI et XVII siècles, Paris, IES, 1991, p. 65.

(45) Henri Jean Victor de SAINT-SimOn, op. cit., p. 246. 


\section{Troupes ennemies et population civile victime des guerres}

L'image des troupes et armées ennemies est présente, quoique pas très abondamment, dans les notes des Français. Choderlos de Laclos profite bien de sa présence à Tilsit en été 1807 pour observer les Russes et noter ses impressions. D'abord, il décrit la garde de l'empereur russe : sa tenue et son comportement au moment du passage de ses supérieurs. Ensuite, Choderlos n'hésite pas à s'arrêter « longtemps » pour « examiner à son aise » l'empereur russe Alexandre $\mathrm{I}^{\text {er }}$. « Il est grand et bel homme, figure distinguée, blond, la taille forte. Pendant que je l'examinais, il lisait un papier qu'il déchira avec lenteur. Sa physionomie exprimait de l'indifférence. Alors il prit une lorgnette pour m'examiner à son tour $»^{46}$. Quelques lignes plus loin, le témoin français fournit l'impression que lui a inspirée le grand-duc Constantin - le frère de l'empereur ${ }^{47}$. Ensuite, il va observer le camp russe sur le bord opposé du Niémen. Enfin, le soir de la même journée, Choderlos entreprend une tentative réussie pour traverser le fleuve afin de visiter le camp russe de plus près. Il décrit « l'aspect sauvage et barbare » des « fameux Kalmuks » en signalant que « ces troupes n'ont point encore donné contre les Français » et que, par conséquent, il « ignore s'ils sont aussi braves que les Cosaques $»^{48}$. Enfin, il est satisfait de rencontrer un officier des troupes régulières et de s'entretenir avec lui en français. Notons que, malgré les difficultés de la campagne de 1812, les Français font preuve d'une attitude équitable et accordent à leurs adversaires des qualités militaires : avant de se replier les troupes russes se défendent avec acharnement et opiniâtreté. Les troupes prussiennes ou les armées autrichiennes - les ennemis mieux connus - ne sont pas décrites dans les textes français.

Enfin, il est important de noter que l'image de la population civile victime des guerres incessantes de cette époque - est régulièrement évoquée dans les récits des héros napoléoniens. En avouant tout leur enthousiasme inspiré par la gloire des armes françaises, ils restent lucides et constatent que la guerre n'est pas le vœu de la majorité de la population qui en souffre énormément. Les réflexions de ce genre sont nombreuses dans la plupart des textes analysés avec, toutefois, une moindre présence dans les écrits datés de l'année 1812. L'inconnu de l'« ailleurs » russe et le déroulement

(46) Étienne Fargeau CHODERLOS DE LACLOS, op. cit., p. 121

(47) Voir également les informations sur Constantin : Maurice de TASCHER, op. cit., p. 52.

(48) Étienne Fargeau CHOdERLOS DE LACLOS, op. cit., p. 122-123. 
stupéfiant de la campagne avaient décidément brouillé les repères des militaires français.

Cette étude montre une fois de plus que l'expérience de mobilité des soldats et des officiers des armées napoléoniennes dans le cadre des opérations militaires pouvait engendrer des pratiques de voyageur, l'écriture des textes descriptifs en étant l'une des conditions nécessaires. En effet, « c'est la manière de vivre et de penser la mobilité, d'introduire l'observation à travers la sécheresse des étapes et des faits, d'arriver à la rencontre de l'autre qui est déterminante pour une définition opératoire du voyage $»^{49}$. Les conquérants napoléoniens font, en effet, preuve d'une curiosité et d'une capacité d'observation exemplaires surtout si l'on prend en compte les conditions très spécifiques de leur mobilité. Leurs écrits ne l'illustrent que trop clairement : les descriptions de la beauté de la nature et des constructions humaines sont insérées parmi les récits des événements militaires et les images terrifiantes de la guerre.

Une large utilisation des documents relatant la campagne de Russie nous a permis d'effectuer une analyse comparative des impressions françaises de l'ensemble des campagnes impériales. Cette approche contribue à effacer certaines tendances à séparer les études des campagnes « victorieuses » des travaux consacrés à la guerre de 1812. En effet, cette dernière est le plus souvent analysée à part ou, tout au plus, dans une optique de comparaison avec la guerre d'Espagne. Cette approche provient d'un regard sur les campagnes napoléoniennes axé sur leur déroulement et leurs résultats. Il semble, cependant, qu'afin d'apporter de nouveaux éclairages sur les mentalités de l'époque, il est temps de (re)penser les campagnes de Napoléon dans leur ensemble, autrement dit de tenter de les voir avec l'œil de leurs contemporains dans leur continuité. Cette étude est une première contribution dans cette direction.

Maya GoubINA

CRLV, Paris IV, CRHS, Paris I

9, rue Friant, 75014, Paris

mgoubina@gmail.com

(49) Nicolas BouRguinAt, « Présentation. Un temps de rupture dans l'histoire des pratiques du voyage » dans Nicolas BOURGUINAT, Sylvain VENAYRE (dir.) op. cit., p. 10. 